Moliner García, O.; Moliner Miravet, L. y Sanahuja Ribés, A. (2018). Percepciones sobre el concepto y la gestión de la diversidad en un centro en proceso de democratización escolar. Revista de Investigación Educativa, 36(2), 455-469. DOI: http://dx.doi.org/10.6018/rie.36.2.293191

\title{
Percepciones sobre el concepto y la gestión de la diversidad en un centro en proceso de democratización escolar
}

\author{
Perceptions about concept and management of diversity in a \\ school in a democratization process
}

\author{
Odet Moliner García, Lidón Moliner Miravet,Aida Sanahuja Ribés \\ Universitat Jaume I de Castellón. Departamento de Pedagogía y Didáctica \\ de las Ciencias Sociales, la Lengua y la Literatura
}

\begin{abstract}
Resumen
El objetivo de este trabajo es analizar la concepción de la diversidad y los procesos de gestión de la misma en un centro inmerso en un proyecto de democratización escolar, desde las percepciones de los actores implicados. A través de un estudio de caso nos centramos en el análisis de las prácticas educativas desarrolladas en un colegio rural agrupado de la provincia de Valencia. La recogida de la información se ha realizado mediante observaciones de aula, entrevistas y diarios de las sesiones. El análisis de la información se ha llevado a cabo mediante análisis de contenido temático. Los resultados ponen de manifiesto las diferentes representaciones de la diversidad de los miembros de la comunidad educativa. Entre las estrategias de gestión de la diversidad utilizadas en las aulas desde un enfoque inclusivo y democrático se destacan el apoyo directo, la graduación de la dificultad de las tareas, la coenseñanza y la ayuda entre iguales. De las prácticas de centro, como el consejo escolar ampliado, se da cuenta de los beneficios y dificultades encontrados y en las conclusiones se ponen en relación las premisas que facilitan los procesos de democratización que tienen en cuenta la diversidad y su manejo efectivo.

Palabras clave: diversidad; democratización; prácticas inclusivas; educación inclusiva.

Correspondencia: Odet Moliner García. Departamento de Pedagogía y Didáctica de las Ciencias Sociales, la Lengua y la Literatura, Universitat Jaume I de Castellón, España.

Proyecto financiado en el que se enmarcó la investigación: “La escuela incluida en el territorio. Análisis de las estrategias de participación ciudadana desde la educación intercultural inclusiva”. MINECO (EDU2013-46491-R).
\end{abstract}




\begin{abstract}
The aim of this work is to analyze the conception of diversity and its management in a center immersed in a school democratization project, by the perceptions of the actors involved. Through a case study we focus on the analysis of educational practices developed in a rural school in the province of Valencia. The data collection has been made through classroom observations, interviews and session diaries. A thematic content analysis has been carried out for data analysis. The results show how a variety of opinions exist regarding diversity by the school community members. Among the diversity management strategies used in the classrooms from an inclusive and democratic approach, some of them are highlighted such as direct support, task difficulty ranking, co-teaching or peer support. From the centre practices, such as the expanded commission, benefits and difficulties have been shown. Conclusions are related to the premises that facilitate democratization process taking into account diversity and their effective management.

Keywords: diversity; democratization; inclusive practices; inclusive education.
\end{abstract}

\title{
Introducción
}

\section{La diversidad y sus múltiples representaciones}

La diversidad del alumnado es una realidad y un fiel reflejo de la sociedad en la que vivimos. Esta diversidad, inherente a la naturaleza humana, abarca gran cantidad de aspectos referidos al género, edad, cultura, religión, orígenes económicos y sociales, expectativas, capacidades, ritmos, intereses y opiniones (Alegre de la Rosa, 2000; Arnaiz, 2005; Jiménez \& Vila, 1999; Stulberg \& Weinberg, 2011). Las formas diferentes de entender y utilizar la palabra "diversidad" plantean desafíos en la búsqueda de la justicia social en el ámbito educativo (Tharp, 2015) ante las cuales se suelen adoptar dos posturas: plantearla como un problema o como un valor y una oportunidad para el aprendizaje. Desde presupuestos inclusivos, en concordancia con la segunda, incidimos en el valor de las diferencias y su aprovechamiento como un estímulo para fomentar el aprendizaje de todos (Ainscow \& Miles, 2008; Booth \& Ainscow, 2000; Echeita, 2010). En la misma línea, Stainback \& Stainback (1999), conciben la diversidad como una característica básica de las aulas inclusivas y confían en que en ellas todos pueden aprender. Por ello, una de las finalidades de la educación inclusiva es reconocer y valorar las diferencias presentes en el alumnado y desarrollar actitudes de aceptación y valoración de la diversidad (Duk \& Narvarte, 2008). Del mismo modo, desde presupuestos interculturales inclusivos, consideramos que valorar la diversidad y respetar la diferencia, es un elemento dinamizador y enriquecedor en la interacción entre personas y grupos humanos (Sales, 2010).

La diversidad tiene un carácter dinámico y abierto, pero, ¿son todas las diversidades iguales? En realidad, no todos los factores de diversidad requieren las mismas respuestas educativas, puesto que mientras algunos deben potenciarse, otros hay que compensarlos (Aldámiz-Echevarría et al. 2009). Aquellos factores que hay que potenciar son los que se derivan, por ejemplo, de distintos valores culturales, de diferentes motivaciones o capacidades del alumnado. En este caso la diversidad equivale a singularidad, y por tanto hay que fomentarla. Por otra parte, los factores a compensar para que no generen desigualdad hacen referencia, por ejemplo, a las necesidades especiales de 
un alumno, ritmos de aprendizaje o procesos de desarrollo desiguales. Por ello, hay que crear espacios que potencien la igualdad de oportunidades para todos a partir de la deconstrucción y reconstrucción de nuestras expectativas culturales y académicas (Braunsteiner \& Mariano-Lapidus, 2014). Y para ello es necesario clarificar los conceptos básicos relacionados con la diversidad, como son la diferencia y la desigualdad. La diferencia, según López Melero (1997) es la apreciación subjetiva, la valoración de la diversidad. Esta valoración puede llevar tras de sí actitudes de rechazo o de aceptación y comprensión. El derecho a la diferencia, es decir, el derecho de cada individuo y de cada comunidad a creerse diferente de los otros, y dotar de contenidos esa voluntad de diferenciación, supone el derecho a la igualdad, es decir, el derecho de ser aceptados como son, a ser indistinguibles ante la lucha por la justicia (Delgado, 1998). Por su parte, la desigualdad establece jerarquías entre personas en función de criterios tales como el poder social, económico, político, de género o étnico. Así pues, lo diverso, en condiciones de igualdad, es deseable, pero la desigualdad tiene que ser corregida si hemos de admitir la universalidad del derecho a la educación (Sales, 2010).

Así pues, consideramos que las concepciones y actitudes sobre la diversidad van a configurar las representaciones sobre la misma y en función del significado que se le atribuya, como valor o como problema, se organizarán las respuestas e interacciones en el contexto educativo.

\section{La gestión de la diversidad y los procesos de democratización}

Afortunadamente, los modelos que educan en la diversidad han ido sustituyendo paulatinamente a los modelos segregadores que conciben la diversidad como un déficit. Tal y como indica Arnaiz (2005), educar para y en la diversidad pasa por ejercer la equidad e igualdad en el contexto escolar, lo que requiere de unas estrategias de enseñanzaaprendizaje que garantice una educación para todos. Continuando con la misma autora:

Educar para la diversidad expresa el matiz de educar para una convivencia democrática donde la solidaridad, la tolerancia y la cooperación estén presentes y caractericen las relaciones entre los alumnos dentro y fuera del aula; se trataría de ser ciudadanos capaces de valorar y vivir con el que es diferente por razones personales, sociales o religiosas (Arnaiz, 2005, p.52).

Para desarrollar este planteamiento, el centro en su totalidad define sus convicciones sociales, culturales y pedagógicas, es decir, explicita cuáles son sus valores y metas, para elaborar una propuesta de actuación coherente con lo expresado. En palabras de González (2008), la diversidad y la inclusión son una cuestión que compete a todo el centro y pasa por observar los principios y valores que orientan y guían al centro y sus prácticas. Dichos aspectos pueden resultar ser facilitadores o barreras que entorpecen el proceso de inclusión. La preocupación por la escuela inclusiva se ha convertido pues en uno de los mayores retos que actualmente deben afrontar los sistemas educativos, los centros, el profesorado y la sociedad (Duran \& Giné, 2011).

Los centros inclusivos asumen un concepto de diversidad amplio y organizan la gestión de la diversidad de manera colaborativa, lo que exige implicación y partici- 
pación de los diferentes miembros de la comunidad escolar en el establecimiento de las metas y proyectos de centro (Moriña \& Parrilla, 2004). En este sentido, el modelo inclusivo se vincula al de escuela democrática (Apple \& Beane, 1999) puesto que ambos conciben la diversidad como un elemento dinamizador de las interacciones humanas y además, según Feito \& López (2008) coinciden en algunos factores como: la creación de condiciones necesarias para que todo el alumnado avance hacia el éxito educativo, la democratización de las aulas para la inclusión de las voces de los niños y adolescentes y la participación de la comunidad educativa en el control y gestión de los centros.

Así pues, consideramos que los centros inmersos en un proceso de construcción de escuela democrática, participativa, intercultural e inclusiva, comparten una visión amplia y no problemática de la diversidad. Y en ellos, la gestión de la diversidad se materializa tanto en estrategias y propuestas metodológicas de gestión de aula que plantean un currículum más democrático (accesible y participativo) como en estrategias de gestión democrática del centro que suponen la ampliación de las voces en la toma de decisiones, así como la implicación de todos los actores educativos en la revisión de las prácticas.

\section{Método}

En este estudio se ha empleado una metodología cualitativa, propia del paradigma interpretativo (Sandín, 2003; Tójar, 2006). Se ha optado por un diseño de estudio de caso en el que se ha tratado de conocer en profundidad el fenómeno investigado. El estudio de caso se centra en la particularidad, la complejidad, la singularidad y la exclusividad de un contexto (Simons, 2011; Stake, 1998). Por ello exploramos un centro en concreto, una escuela inmersa en un proyecto amplio y complejo de transformación escolar que está incorporando prácticas inclusivas y democratizadoras.

\section{Objetivos}

Los objetivos de esta investigación son dos:

a) Analizar cuál es la concepción de la diversidad que tienen los diferentes agentes educativos y a qué factores la asocian, viendo si la consideran un valor o un problema.

b) Describir las percepciones de los actores implicados sobre cómo se gestiona la diversidad en el centro y las propuestas que se realizan para conseguir un currículum más democrático y participativo.

\section{Población y Muestra}

La investigación se desarrolla en un CRA (Centro Rural Agrupado) de la provincia de Valencia que consta de dos aularios ubicados en pueblos vecinos. El centro cuenta con 150 alumnos y 15 profesores, y se trata de un centro innovador, con un uso considerable de recursos TIC. Es el cuarto año que desarrolla un proyecto para la construcción de la escuela intercultural, inclusiva y democrática mediante procesos de investigación-acción-participativa. En el centro se utilizan metodologías activas y participativas como la lectura en parejas (Moliner, Moliner, Sanahuja \& Sanmateo, 2015), el aprendizaje basado en proyectos, los rincones (Benet, Sanahuja \& Moliner, 2016), así 
como el trabajo cooperativo. Se desarrollan diversas estrategias de visibilización del centro y proyectos de vinculación con el territorio (Moliner, Sales \& Sanahuja, 2017; Sales, Traver, Sanahuja \& Benet, 2017), así como estrategias de participación democrática, incorporando la autogestión mediante comisiones mixtas de trabajo y el consejo escolar ampliado como órgano de toma de decisiones colectivas.

El estudio de caso se ha focalizado en el análisis de las prácticas educativas desarrolladas en una clase de infantil y siete clases de primaria, algunas de ellas multigrado (agrupadas por ciclos), ubicadas en dos aularios. Se ha recogido la información generada a partir de un proceso de revisión de cinco prácticas y los participantes en el estudio, distribuidos en función de la práctica analizada (Ver Tabla 1).

Tabla 1

Participantes

\begin{tabular}{|c|c|c|c|}
\hline $\begin{array}{l}\text { Prácticas } \\
\text { revisadas }\end{array}$ & Aulas & $\begin{array}{c}\text { Edad } \\
\text { alumnado }\end{array}$ & Participantes \\
\hline $\begin{array}{l}\text { Lectura en } \\
\text { pareja (LP) }\end{array}$ & $\begin{array}{l}\text { Aulario A: } 2^{\circ}, 3^{\circ} \\
\text { y } 4^{\circ} \\
\text { Aulario B: } 3^{\circ}-4^{o} \text { y } \\
5^{o} \text { y } 6^{\circ}\end{array}$ & 7 a 12 & $\begin{array}{l}3 \text { observadores externos: registro narrativo } \\
6 \text { madres (observación en el aula y entrevistas) } \\
9 \text { docentes entrevistados (maestros tutores } \\
\text { y maestro de apoyo, docentes que realizan } \\
\text { tareas de apoyo no siendo de pedagogía } \\
\text { terapéutica o audición y lenguaje) } \\
10 \text { alumnos entrevistados ( } 2 \text { por cada aula) }\end{array}$ \\
\hline Rincones (R) & $\begin{array}{l}\text { 1r ciclo de } \\
\text { primaria }\left(1^{\mathrm{o}} \text { y } 2^{\underline{o}}\right)\end{array}$ & 6 a 8 & $\begin{array}{l}3 \text { observadores externos: registro narrativo } \\
1 \text { madre y } 1 \text { padre (observaciones en el aula y } \\
\text { posterior entrevista) } \\
2 \text { alumnos entrevistados } \\
1 \text { docente tutor entrevistado }\end{array}$ \\
\hline $\begin{array}{l}\text { Puzle Aronson } \\
\text { (PA) }\end{array}$ & $\begin{array}{l}\text { 3r ciclo de } \\
\text { primaria }\left(5^{\circ} \text { y } 6^{\circ}\right)\end{array}$ & 10 a 12 & $\begin{array}{l}3 \text { observadores externos: registro narrativo } \\
2 \text { madres (observaciones en el aula y posterior } \\
\text { entrevista) } \\
2 \text { alumnos entrevistados } \\
2 \text { docentes entrevistados } \\
1 \text { tutora y } 1 \text { maestra de apoyo entrevistadas. }\end{array}$ \\
\hline $\begin{array}{l}\text { Lápices al } \\
\text { centro (LC) }\end{array}$ & Infantil & 5 & $\begin{array}{l}3 \text { observadores externos: registro narrativo } \\
2 \text { madres (observaciones en el aula y posterior } \\
\text { entrevista) } \\
2 \text { alumnos entrevistados } \\
1 \text { docente tutor entrevistado }\end{array}$ \\
\hline $\begin{array}{l}\text { Consejo escolar } \\
\text { ampliado (CO) }\end{array}$ & $\begin{array}{l}\text { Claustro de } \\
\text { profesores, } \\
\text { alumnos, familias } \\
\text { y agentes } \\
\text { comunitarios }\end{array}$ & varias & $\begin{array}{l}3 \text { alumnos entrevistados } \\
2 \text { maestros entrevistados }\end{array}$ \\
\hline
\end{tabular}




\section{Instrumentos y procedimiento de recogida de la información}

La recogida de la información se ha realizado a través de observaciones de aula, entrevistas y diarios (registros narrativos) de las sesiones de clase, con el objetivo de triangular los datos y las fuentes, puesto que tanto en las entrevistas como en las observaciones participaron estudiantes, profesorado, familias e investigadores (como observadores externos).

Las observaciones de las prácticas de aula han permitido la descripción sistemática de eventos y comportamientos en el escenario social elegido para ser estudiado (Marshall \& Rossman, 1989). Los instrumentos para la recogida de datos fueron registros narrativos y fichas de observación tipo hojas de comprobación o check-list utilizadas por los observadores externos (investigadores) y las familias.

Las entrevistas sirvieron para recoger información de manera sistemática con maestros y maestras, familias y alumnado, con el objeto de contrastar y/o ampliar información sobre sus percepciones sobre las prácticas observadas (Buendía, Colás \& Hernández, 1998). Como instrumentos se manejaron 3 guiones de entrevista semiestructurada: uno para el alumnado, otro para las familias y un tercero para los docentes.

Tanto las fichas de observación como los guiones de las entrevistas se elaboraron a partir de los factores de la dimensión C. Cómo aprendemos y enseñamos de la Guía CEIN (Sales, Moliner \& Traver, 2010), una herramienta que ayuda a orientar la exploración y el diagnóstico contextual de la realidad escolar y educativa desde un enfoque intercultural e inclusivo.

\section{Procedimiento de análisis de datos}

Recogida toda la información se pasó a la transcripción literal de los datos para realizar posteriormente un análisis de contenido temático. El pre-análisis, codificación y establecimiento de categorías se hizo por el equipo investigador para garantizar la fiabilidad de las mismas a través del acuerdo intersubjetivo entre codificadores. La validación de la definición de las categorías se realizó por juicio de expertos (10 jueces de universidades españolas) en el marco de la investigación general de la que forma parte este trabajo. La categoría analizada se denominó inicialmente "reconocimiento y valoración de la diversidad" y los datos empíricos categorizados fueron agrupados por analogía según criterios de pertenencia y congruencia. La categorización se realizó a través del ATLAS.ti para el análisis de contenido cualitativo y CmapTools para la representación gráfica del mapa relacional.

A continuación, en la Tabla 2, presentamos el sistema de codificación que se utiliza para identificar la procedencia de la cita.

Tabla 2

Sistema de codificación

\begin{tabular}{lll}
\hline \multicolumn{1}{c}{ Práctica } & \multicolumn{1}{c}{ Instrumento } & \multicolumn{1}{c}{ Informante } \\
\hline Lectura en pareja (LP) & Entrevista (E) & Alumno/a (A) \\
Rincones (R) & Observación (O) & Profesor/a (P) \\
Puzle Aronson (PA) & Diario de la sesión (DS) & Familia (F) \\
Lápices al Centro (LC) & & Grupo de investigación (M) \\
Consejo escolar ampliado (CO) & & \\
\hline
\end{tabular}




\section{Resultados}

Los resultados se presentan tomando como referencia las dos dimensiones del estudio: 1) conceptualización de la diversidad y 2) gestión de la diversidad y propuestas de mejora en relación con el proceso de democratización escolar.

\section{Conceptualización de la diversidad}

Seguidamente, en la Figura 1, se presenta el mapa relacional sobre la conceptualización de la diversidad según los implicados.

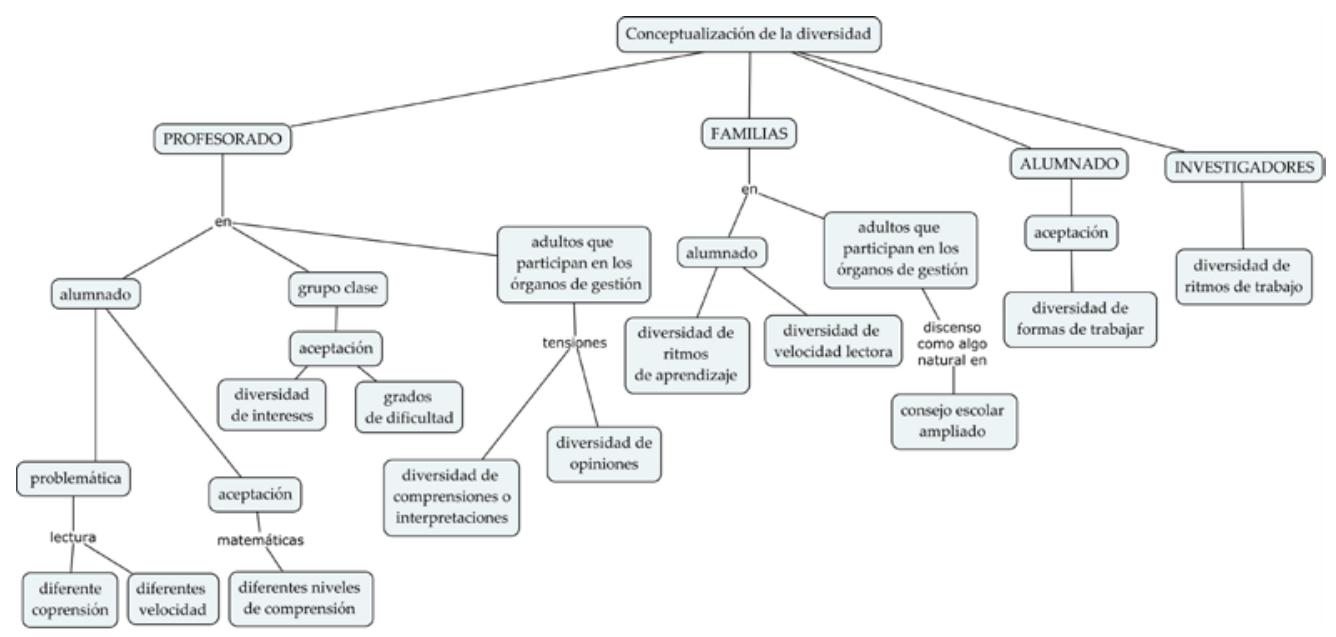

Figura 1. Mapa relacional sobre la conceptualización de la diversidad

a) El profesorado percibe la diversidad del alumnado, del grupo clase y de los adultos que participan en los órganos de gestión. Cuando se refiere a las prácticas de aula tienen una visión de la diversidad del alumnado como problema y aluden a factores de velocidad y comprensión lectora: "Yo en este caso las hago por velocidad lectora y, también un poco, mirando los problemas que tienen en la comprensión. (LP, E, P3); “[...] de esa manera, si veo que hay uno que tenga más problema lo pongo con alguien que pueda tener el mismo problema. La manera de que se ayuden partiendo del mismo nivel, digamos el mismo problema ellos se refuerzan y es una manera de darles más importancia y confianza. $(L P, E, P 3)^{\prime \prime}$. Otros aceptan las diferencias entre alumnos como algo natural: diferentes niveles de comprensión en matemáticas: "Porque si cojo a los más buenos, en dos minutos han terminado. Sólo tengo que preguntar una vez cada pregunta. Entonces yo intento que haya gente que le cuesta un pelín más el cálculo y que va más sobrada. (LC, E, P6)".

Reconocen las diferencias entre los grupos -clase: diversidad de intereses y de grados de dificultad: "Cada grupo tiene unas características diferentes. No valen el mismo tipo de lecturas, hay lecturas que funcionan mejor en un grupo, la extensión, el vocabulario en algún momento que se tiene que adaptar depende del grupo. (LP, E, P3)" 
Entre las personas que participan en el consejo, perciben la diversidad de comprensiones o interpretaciones de una misma situación: "Yo lo que sí que creo es que a veces, dentro de la comisión, cuando se ha hablado algún tema, alguna persona no lo ha entendido bien y no ha recalcado sobre el tema. O no le ha parecido bien algo y no ha recalcado 'a mí no me parece bien'. (CO, E, P9)". También se detecta una diversidad de opiniones que puede generar tensión: "Yo creo que hay muy buen rollo, aunque a la gente a veces te pilla en un punto en que contestas de una manera que no... no te gustaría contestar, y después pides disculpas y ya está, porque hay momentos tensos realmente...Porque cuando ves a una persona que se empeña en algo y que no es capaz de ser reflexivo, tal vez como tú eres más reflexivo dices: 'jostras tú! os estamos dando una oportunidad de ser reflexivos, pero no lo estás siendo' ...Y después lo que he dicho antes, es cuestión de aprendizaje, yo pienso que esos momentos de tensión cada vez serán menos si la gente aprende a participar. (CO, E, P6)". Sin embargo, aunque diferentes opiniones generan tensión, consideran que a ser reflexivo y a participar se aprende.

b) Las familias perciben la diversidad de ritmos de aprendizaje del alumnado "[...] la madre, valora muy positivamente la práctica porque cómo ella dice, respeta los diferentes ritmos de aprendizaje, no tienen que hacerlo todos a la vez, sino que cada cual trae su ritmo. $(R, D S, F 3)$ " y la diversidad de velocidad lectora: "Diferentes niveles ¿no?, que unos leen más rápido (LP, E, F2)". Valoran la unión y el trato igualitario entre el alumnado: "No hay ningún tipo de diferencia entre los niños, allí se llama lo que diríamos en general niños, todos iguales. Me agrada como se tratan los niños entre ellos, no hay ninguna discriminación de ninguna clase. Están todos muy unidos ( $R, E, F 1)$ ". Así como los valores que se fomentan como el respeto entre iguales. También perciben la diversidad de opiniones entre los participantes en el consejo escolar y consideran el disenso como algo natural: "Pero sí se suele escuchar a unos y a otros. Cuando hay un acuerdo, perfecto. $Y$, si no hay un acuerdo, pues no siempre podemos estar todos de acuerdo, esto forma parte de la vida. (CO, E, F3)".

c) Los alumnos perciben la diversidad en sus compañeros de formas de "hacer" o de trabajar. Cuando se les pregunta sobre el cambio de parejas en la tutoría entre iguales ellos afirman que les gusta porque así pueden interaccionar con otros compañeros. "Porque conocemos como trabaja más gente. (LP, E, A1)"

d) Los investigadores se refieren a la diversidad de ritmos de trabajo en el aula: "Los primeros acaban a las 9:25 y a las 9:30 quedan dos parejas trabajando. (LP, O, M)"

\section{Gestión de la diversidad y propuestas de mejora}

En el centro, para dar respuesta a la diversidad se desarrollan diversas prácticas curriculares que responden a la idea de diversificación (Tomlinson, 2003) pues se combinan diferentes agrupamientos del alumnado en función de los objetivos previstos, incluyendo actividades que se estructuran de manera cooperativa y favorecen la ayuda mutua (lectura en parejas, puzle de Aronson o lápices al centro); que favorecen diferentes ritmos de aprendizaje e intereses (rincones) y que propician la participación democrática (los estudiantes participan en el consejo escolar ampliado). 
Las actividades organizadas de manera cooperativa (lectura en pareja, puzle y lápices al centro) aunque son las mismas para todos los estudiantes, estos pueden recibir diferentes tipos y fuentes de apoyo en función de sus necesidades. Las estrategias utilizadas son:

a) apoyo más directo: "Algún estudiante que lo necesita recibe un apoyo más directo, por ejemplo, haciendo pareja con la maestra: Hace lo mismo, una semana es el tutor y la otra el tutorado. Entonces cuando yo o el otro maestro de refuerzo somos el tutor le refuerzan las diferentes etapas de la lectura por parejas al mismo tiempo hacemos un modelo de la manera de leer, la lectura y todo. (LP, E, P3)". "También le hacemos el refuerzo sólo a él. En esta técnica no hay refuerzo entre iguales, sólo lo hacemos los docentes. (PZ, E, P7)"

b) graduación de la dificultad de las tareas y/o preguntas: "Intentamos que el problema sea un poco más fácil. De los cuatro problemas le asignamos el más sencillo. (PZ, $E, P 2)^{\prime \prime}$. El profesorado propone trabajo colaborativo para coordinar criterios de selección de lecturas por ciclos, para responder a los diferentes niveles: " $M$. introduce que un punto importante a tratar sería el de preparar/seleccionar las lecturas (tipos, preguntas,...) conjuntamente todo el ciclo. Habría que establecer unos criterios de selección. Comentan también de graduarlas por nivel de dificultad o progresión. (LP, DS, P4)" e intereses: "V. apunta la necesidad de ajustar la dificultad de lecturas y las temáticas. Poco a poco van mejorándose la propuesta para que sea atractiva al tiempo que logra la comprensión y velocidad lectora. (LP, DS, P6)"

c) dos maestros en el aula: 'Cómo somos impares, $M$. que entra a hacer el refuerzo se ocupa. En el primer semestre era uno y ahora es otro. (LP, E, P4)"

d) diversificación del espacio: "[...] en 3 r ciclo por número, porque es muy numeroso, porque si no se arma mucho de alboroto. Vamos unos cuántos al aula de informática y ya no es tan... ruidoso. (LP, E, P1)" y el tiempo: "Normalmente, si acaban antes pues siempre hacen alguna actividad o algo... sobre todo los mayores alguna cuestión que tengan que acabar de la clase para no cortar y continuar con otra cosa. Para que los tres cuartos sí que se utilicen. (LP, E, P1)". "Los primeros acaban a las 9:25 y a las 9:30 quedan dos parejas trabajando. Los que acaban cogen un libro de lectura (LP, $O, M)^{\prime \prime}$.

e) ayuda entre iguales:

- homogénea: formando parejas del mismo nivel de competencia: "Yo entiendo que si hay un niño al que le cuesta mucho leer con uno que enseguida lo hace, perderá interés uno o el otro (LP, E, F2)". "No, porque también tienen a uno de su nivel entonces se tienen que potenciar entre los dos. Porque después volvemos a lo mismo, después en clase leen todos. ( $L P, E, F 2)^{\prime \prime}$. Las familias ven bien que se formen las parejas del mismo nivel.

- heterogénea: "Porque si cojo a los más buenos, en dos minutos han terminado. Sólo tengo que preguntar una vez cada pregunta. Entonces yo intento que haya gente que le cuesta un pelín más el cálculo y que va más sobrada (LC, E, P6) ". "Cuando se juntan por especialistas y corrigen el mismo problema los compañeros se ayudan entre ellos, en el caso de que no lo entiendan. (PZ, E, P7)"

f) adaptación de la autoevaluación: "Lo único que va cambiando es la evaluación, el tema de la autoevaluación. En los niños de 1r ciclo es más sencilla y después, a lo mejor va subiendo de nivel y cada tutor se coge un poquito los ítems que piensa que son más adecuados. Ahí sí que puede ser que haya algún cambio en las pautas de la evaluación. (LP, E, P1)". 
Por otra parte, se utilizan los rincones para favorecer el aprendizaje autónomo del alumnado, lo cual permite gestionar:

- los diferentes ritmos de trabajo y de aprendizaje: "A mí me ha parecido bien el tema de la metodología, estaban cada uno dentro de su nivel pues haciéndolo... sin prisa, dedicando el tiempo que cada uno estimara oportuno para hacer la actividad. $(R$, E, F1)" "A mí también me ha gustado mucho que cada uno sabía lo que tenía que hacer a su ritmo, a su manera y sin prisas. Y me ha gustado mucho esa manera que tiene $J$. el profesor de que todos hagan toda clase de niveles, tanto lingüístico,... (R, E, F2)". "Casi todos han hecho entre 3 y 4 rincones cuando acaba la sesión, antes del patio. $R$. ha hecho 5. ( $R, O, M)$ " "[...] la madre, valora muy positivamente la práctica porque cómo ella dice, respeta los diferentes ritmos de aprendizaje, no tienen que hacer todos lo mismo a la vez, sino que cada cual va a su ritmo. ( $R, D S, F 3)$ "

- los diferentes intereses y motivaciones: "Si todos han de hacer lo mismo, al mismo tiempo, el que puede ir más no va, otros se aburren y otros no llegan a lo mejor a la presión de lo que estás pidiendo. ( $R, E, P 8)$ ". "Trabajar por rincones favorece el trabajo autónomo y que cada cual pueda mantener su ritmo de aprendizaje, sus inquietudes. Y cuando se agobien de matemáticas no tengo ningún problema de aguantar los tres cuartos de hora que dice la ley, ellos pueden cambiar y estar con la tablet, por ejemplo. En el mismo tiempo un niño puede haber hecho una ficha, estar con la tablet, leer un libro... ( $R, E, P 8)$.

- los diferentes niveles de competencia curricular: los investigadores proponen la utilización de códigos visuales en función de nivel de dificultad de las actividades para favorecer el seguimiento del progreso del alumnado: “...propone que utilicen un código de colores de más fácil a más difícil, que sea muy visual y que permita elegir y hacer el seguimiento en las diferentes actividades. Tanto a la madre como a J. les parece una buena propuesta. ( $R, D S, M)^{\prime \prime}$

En cuanto a la práctica del consejo escolar ampliado, surge con la idea de favorecer la participación democrática de más personas en la toma de decisiones. Las familias y alumnado apuntan factores imprescindibles como:

a) la escucha activa y la comunicación positiva: "Siempre en positivo. Incluso la manera de decir las cosas es muy buena. Cuando alguien tiene un reproche que hacer no viene 'ggggg' [sonido que imita el enfado], no. 'Escucha que aquello de..., mira que... podríais hacerlo de otro modo' (CO, E, F3)".

b) la posibilidad de expresar ideas: 'A mí me ha gustado mucho porque puedes expresar tus ideas y puedes contar a los demás todo lo que hacemos y lo que pensamos, sí...(CO, E, A3)"

Aunque por otra parte, el profesorado, estando de acuerdo con la estrategia general de ampliar las voces, percibe como una dificultad la gestión de la información y la diversidad de opiniones y expresan:

a) una percepción negativa sobre la valoración de la información compartida con las familias: "La comisión...lo digo por la situación. Yo creo que... intentamos transmitir toda la información, toda, que no se nos escape nada, pero tampoco se valora. No creo que 
se esté valorando todo lo que se está transmitiendo, todo lo que se está dando a opinar, todo lo que se hace partícipe a los demás". (CO, E, P9) y falta de reconocimiento del trabajo: "Y yo no disfruto... cuando toca reunirse la comisión, no estoy a gusto, porque sé que a la mínima cosa que se propone siempre hay uno 'pero'. Nunca es ';Ah! qué bueno que ha sido esto', no, siempre hay 'peros, peros y peros'.(CO, E, P9)

b) la idea de que las familias son más eficaces en la acción que en la reflexión: “Cualquier actividad, están ahí los primeros, y son una pasada. O sea, que no hay, pero... están ofuscados en cualquier cosa ahora. (CO, E, P9)".

Por último, el alumnado reclama su espacio en las sesiones del consejo ampliado: "hay demasiada gente y no podemos participar todos" (CO, E, A3); "de alumnos no hay tantos" (CO, E, A4 ); "Yo modificaría cuando hablan los mayores que dejaran hablar también a los niños, que no estén todo el rato hablando ellos, que dejaran que un niño por ejemplo diga 'pues tal, tal y tal tal'... no sé ... me gustaría que hablaran más los niños. "(CO, E, A2).

\section{Discusión y conclusiones}

En cuanto a las percepciones de la diversidad por parte del profesorado, se observa cómo existe una dualidad de opiniones al respecto. Cuando se refieren al alumnado como individuos, por una parte, consideran la diversidad como un problema y por otra como algo natural. Si nos detenemos en el grupo clase, vemos cómo relacionan la diversidad con los diferentes intereses y motivaciones del alumnado. Al mismo tiempo consideran la necesidad de crear materiales acordes con sus competencias y habilidades para adaptarse a las características de todo el alumnado. Esto nos remite al enfoque más "humano" de la diversidad, el cual la concibe como un valor en sí mismo y como una posibilidad de enriquecimiento mutuo. De ahí, de acuerdo con Moriña (2008), rescatamos la necesidad de asentar la formación del profesorado en la reflexión sobre el propio concepto de diversidad, las herramientas para la planificación de los apoyos, las metodologías didácticas y las estrategias organizativas inclusivas. Aunque la heterogeneidad del alumnado es percibida positivamente por los iguales, investigadores y familias, estas últimas se refieren a cuestiones más curriculares (velocidad y comprensión lectora) y de interacción entre iguales, lo cual nos lleva a pensar en los dos temas que preocupan especialmente a las familias: el aprendizaje y la convivencia.

La escuela inclusiva y democrática pone en marcha estrategias que suponen emprender lo que denominaríamos "prácticas curriculares" que vehiculan la gestión de la diversidad desde un enfoque inclusivo. Esto pasa por cuestionar algunas que están instauradas en la mayoría de nuestros centros, por ejemplo, el apoyo directo del profesor o del especialista al alumno/a que más lo necesita. Como alternativa tenemos una forma de organizar y gestionar el aula basada en trabajo de dos maestros en el aula (Huguet, 2006), lo que se traduce en una cultura colaborativa de centro (Meredith et al., 2017), puesto que exige una mayor coordinación entre los docentes a la hora elaborar materiales de aula que permitan responder a los diferentes niveles de aprendizaje del alumnado (Zabalza, 1990). También la ayuda o el apoyo entre iguales (Cowie \& Fernández, 2006; Muntaner, 2014; Topping, Buchs, Duran \& Van Keer, 2017) es otro elemento a destacar en las que el alumnado se convierte en protagonista del proceso 
de enseñanza aprendizaje. Existen diferentes percepciones en cuanto a la agrupación del alumnado en la ayuda entre iguales. Las familias consideran que el nivel de competencias del alumnado debe ser homogéneo para que así no se pierda el nivel y el interés por la tarea a realizar (Moliner, Moliner, Sanahuja \& Sanmateo, 2015). Por su parte, el profesorado plantea la necesidad de la heterogeneidad en prácticas grupales cooperativas como "lapiceros al centro" y "puzzle de Aronson".

Otros elementos que emergen de la investigación, considerados clave en la gestión de la heterogeneidad del aula son: a) la diversificación de tiempos y espacios, de acuerdo con los trabajos de Leroux \& Paré (2016), b) la gradación de las tareas según el nivel de dificultad de las mismas, también referenciado en el trabajo de Ruiz \& Bel (2008) y c) la necesidad de adaptar los procesos de evaluación para que sea más inclusiva y democrática (Murillo \& Hidalgo, 2016). Ello supone una revisión global de la organización y cultura escolar y de las prácticas, no sólo desde cambios coyunturales que pueden resolver situaciones problemáticas inmediatas sino para favorecer una transformación profunda de la institución (Santos, 2006).

Por otra parte, del trabajo se desprende que la gestión del factor de diversidad que genera mayor tensión es la diversidad de opiniones, siendo esta una percepción particular del profesorado, mientras que las familias lo perciben como algo natural. El consejo escolar ampliado como estrategia de democratización permite la expresión de opiniones variadas y contrapuestas sobre temáticas que afectan a la vida del centro. Las familias aceptan que el disenso forma parte de los procesos de participación y democratización de los centros educativos, mientras que el profesorado considera que la falta de comprensión por parte de los asistentes puede llevar a interpretaciones erróneas de la información tratada. Así pues, consideramos de vital importancia establecer un lenguaje común que nos permita avanzar hacia objetivos y valores inclusivos e interculturales compartidos y consensuados por la comunidad educativa (Moliner, Moliner \& Sales, 2010). Ello no es fácil, sobre todo en espacios que tradicionalmente han sido ocupados por el profesorado desde una posición profesionalista. Por ello, la práctica constante del diálogo es, como apunta Santos Guerra (2000), el elemento esencial de la negociación, de la deliberación racional, de procesos dialógicos y cooperativos que permiten la acción conjunta de la comunidad educativa (alumnado, profesorado, familias y agentes sociales y comunitarios).

Sin embargo, pese a los esfuerzos de democratización del centro, la voz del alumnado parece quedar silenciada y relegada a un segundo plano. El alumnado reclama una mayor presencia y participación de las sesiones del consejo ampliado. Coincidiendo con Susinos (2012), es cierto que los consejos escolares pueden representar un espacio idóneo para la participación institucional del alumnado. No obstante, y siguiendo con la misma autora, existen diferentes investigaciones que han puesto en entredicho el papel de los consejos escolares como promotores de la gestión democrática y participativa, puesto que se han constituido como espacios meramente formales, donde el rol del estudiantado ha sido muy secundario. Lo que parece estar claro y hay que seguir reclamando es cómo la voz del estudiantado no solo puede ayudar a aprender, sino también a mejorar el compromiso social y cívico con la escuela y con el entorno (Baroutsis, McGregor \& Mills, 2016).

En un reciente trabajo, Fillion, Bergeron, Prud'homme \& Traver (2016) apuntan algunos de los factores clave para construcción de una escuela inclusiva y democrática, en 
consonancia con los resultados de nuestro estudio como son: 1) los valores democráticos de justicia y equidad, 2) el reconocimiento y la toma en consideración de la diversidad y 3) la participación y el aprendizaje de la convivencia. El proceso de revisión de las prácticas de manera participativa favorece la movilización del conocimiento de los actores implicados y genera nuevas propuestas pedagógicas situadas (en contexto) que van a mejorar las prácticas desarrolladas. Es decir, las propuestas se generan por parte de profesorado, familias, alumnado e investigadores en condición de igualdad, lejos de modelos expertos. Esto favorece que todos los miembros de la comunidad puedan acceder, participar y progresar en el aula inclusiva y de esta manera que se escuchen sus voces y por tanto se movilicen y reconozcan diferentes fuentes de conocimiento y saberes. Y esto en consonancia con el trabajo de Moliner, Traver, Ruiz \& Segarra (2016), que propone siete estrategias que posibilitan la construcción de escuelas democráticas como: 1) propiciar una cultura colaborativa, 2) luchar contra la exclusión y valorar la diversidad, 3) redefinir los valores democráticos, 4) que la comunidad participe y tome decisiones, 5) utilizar la investigación-acción participativa, 6) autoformación sobre participación democrática y 7) desarrollar proyectos compartidos entre el centro educativo y su territorio. De este modo e integrando estas premisas, se facilitan procesos de democratización que tienen en cuenta la diversidad y su gestión efectiva en los centros.

\section{Referencias}

Ainscow, M., \& Miles, S. (2008). Making education for all inclusive: Where next? Prospects, 38(1), 15-34.

Aldámiz-Echevarría, M., Alsinet J., Basssedas, E., Giné, N. Masalles, J., Masip, M., Muñoz, E., Notó, C., Ortega, Á., Ribera, M., \& Rigol, A. (2009). ¿Cómo hacerlo? Propuestas para educar en la diversidad. Barcelona: Graó.

Alegre de la Rosa, O. (2000). Diversidad humana y educación. Málaga: Aljibe.

Apple, M.W. \& Beane, J.A. (1999). Escuelas democráticas. Madrid: Morata.

Arnaiz, P. (2005). Atención a la diversidad. Programación curricular. San José, Costa Rica: EUNED.

Baroutsis, A., McGregor, G. \& Mills, M. (2016). Pedagogic Voices: Student Voices in Teaching and Engagement Pedagogies. Pedagogy, Culture and Society, 24(1), 123-140.

Benet, A., Sanahuja, A. \& Moliner, L. (2016). Rincones, from the teachers' perspectives: a reflection on a primary education practice. 9 th International Conference of Education, Research and Innovation. Sevilla.

Booth, T. \& Ainscow, M. (2000). The Index for Inclusion: Developing Learning and Participation in Schools. London: CSIE.

Braunsteiner, M.L. \& Mariano-Lapidus, S. (2014). A perspective on inclusion: Challenges for the future. Global Education Review, 1 (1). 32-43.

Buendía, L., Colás, P. \& Hernández, F. (1998). Métodos de investigación en psicopedagogía. Madrid: McGraw Hill.

Cowie, H. \& Fernández, F.J. (2006). Ayuda entre Iguales en las escuelas: desarrollo y retos. Revista Electrónica de Investigación Psicoeducativa. (4, 2) 291-310.

Delgado, M. (1998). Diversitat i integració. Barcelona: Empúries. 
Duk, C. \& Narvarte, L. (2008). Evaluar la Calidad de la Respuesta de la Escuela a la Diversidad de Necesidades Educativas de los Estudiantes. Revista Electrónica Iberoamericana sobre Calidad, Eficacia y Cambio en Educación, 6(2), 137-156.

Duran, D. \& Giné, C. (2011). La formación del profesorado para la educación inclusiva: Un proceso de desarrollo profesional y de mejora de los centros para atender la diversidad. Revista Latinoamericana de Educación Inclusiva, 5(2), 153-170.

Echeíta, G. (2010). Alejandra L. S. o el dilema de la inclusión educativa en. España. En: P. Arnaiz, M. D. Hurtado, y F. J. Soto (Coords.) 25 años de integración escolar en España. Tecnologías e inclusión en el ámbito educativo, laboral y comunitario. (pp. 1- 13) Murcia: Consejería de. Educación, Formación y Empleo.

Feito, R. \& López, J.I. (2008). Construyendo escuelas democráticas. Barcelona : Hipatia.

Fillion, P.L., Bergeron, G., Prud'homme, L. \& Traver, J. (2016). L'éducation à la citoyenneté démocratique: un enjeu fondamental associé au projet d'inclusion scolaire et aux pratiques de différenciation pédagogique. Dans L. Prud'homme, H. Duchesne, P. Bonvin, et R. Vienneau (Eds.), L'inclusion scolaire : ses fondements, ses acteurs et ses pratiques. Bruxelles: De Boeck Supérieur.

González, T. (2008). Diversidad e inclusión educativa: algunas reflexiones sobre el liderazgo en el centro escolar. REICE - Revista Electrónica Iberoamericana sobre Calidad, Eficacia y Cambio en Educación, 6 (2), 82-99.

Huguet, T. (2006). Aprendre junts a l'aula. Una proposta inclusiva. Barcelona: Graó.

Jiménez, P. \& Vilá, M. (1999). De la educación especial a la educación en la diversidad. Málaga: Aljibe.

Leroux, M. \& Paré, M. (2016). Mieux répondre aux besoins diversifiés de tous les élèves. Des pistes pour différencier, adapter et modifier son enseignement. Montréal: Chenelière éducation.

López Melero, M. (1997). Escuela pública y atención a la diversidad. La educación intercultural: la diferencia como valor. En AA.VV. Escuela pública y sociedad neoliberal (pp. 15-150). Málaga: Aula libre.

Marshall, C. \& Rossman, G. B. (1989). Designing qualitative research. Newbury Park, : Sage.

Meredith, C., Moolenaar, N. M., Struyve, C., Vandecandelaere, M., Gielen, S., \& Kyndt, E. (2017). The measurement of collaborative culture in secondary schools: An informal subgroup approach. Frontline Learning Research, 5(2), 24-35.

Moliner, L., Moliner, O. \& Sales, A. (2010). Conocer la cultura de un centro y las miradas del profesorado sobre la diversidad sociocultural y personal de la comunidad como punto de arranque para la transformación educativa, Cultura y Educación, 22(3), 283-296.

Moliner, O., Moliner, L., Sanahuja, A. \& Sanmateo, V. (2015). Análisis de los elementos de la tutoría entre iguales que posibilitan avanzar hacia la construcción de una escuela intercultural inclusiva y democrática. Revista latinoamericana de educación inclusiva. 9(2), 41-58.

Moliner, O., Sales, A. \& Sanahuja, A. (2017). Social Mapping in the Context of a Community-build Day: Strategy to Strengthen Links with Community in a Small Rural School. Procedia - Social and Behavioral Sciences, 237, 305-310. Recuperado de http://dx.doi.org/10.1016/j.sbspro.2017.02.083

Moliner, O., Traver, J. A., Ruiz, M. P. \& Segarra, T. (2016). Estrategias que inciden en los procesos de democratización de la escuela. Una aproximación teórica. Revista Electrónica de Investigación Educativa, 18(2), 116-129. Recuperado de http://redie.uabc. $\mathrm{mx} /$ redie/article/view/1110 
Moriña, A. \& Parrilla, A. (2004). Criterios para la formación permanente del profesorado en el marco de la educación inclusiva. Revista de Educación, 339, 517-539.

Moriña, A. (2008). La escuela de la diversidad. Madrid: Síntesis.

Muntaner, J.J. (2014). Prácticas inclusivas en el aula ordinaria. Revista nacional e internacional de educación inclusiva, 7 (1), 63-79.

Murillo, J. \& Hidalgo, N. (2016).Evaluación Democrática y para la Democracia. Revista Iberoamericana de Evaluación Educativa, 9(1), 5-7.

Ruiz \& Bel, R. (2008). Plans múltiples i personalitzats per a l'aula inclusiva. Vic: Eumo.

Sales, A. (2010). La formación continua como proceso clave en la profesionalización docente. Revista latinoamericana de educación inclusiva, 4 (1), 65-82.

Sales, A., Traver, J.A., Benet, A. y Sanahuja, A. (2017). A Community Wellcome day: Cultural Diversity and Critical Citizenship in a Included School. The Mobilities, Transitions, Transformations Intercultural Education at the Crossroads International Conference. Budapest, Hungary.

Sales, A., Moliner, O. \& Traver, J. (Eds.) (2010). La construcción de la escuela intercultural inclusiva desde procesos de investigación-acción. Castellón: Servicio de Publicaciones de la Universitat Jaume I.

Sandín, M. P. (2003). Investigación Cualitativa en Educación. Fundamentos y Tradiciones. Madrid: Mc Graw and Hill.

Santos Guerra, M. A. (2000). La escuela que aprende. Madrid: Morata.

Santos, M. (2006). Participación, democracia y educación: cultura escolar y cultura popular. Revista de Educación, 339, 883-901.

Simons, H. (2011). El estudio de caso: Teoría y práctica. Madrid: Morata.

Stainback, S. \& Stainback, W. (1999). Aulas inclusivas. Madrid: Narcea.

Stake, R.E. (1998). Investigación con estudio de casos. Madrid: Morata.

Stulberg, L. M. \& Weinberg, S. L. (2011). Introduction. In L. M. Stulberg \& S. L. Weinberg (Eds.), Diversity in American higher education: Toward a more comprehensive approach (pp. 1-4). New York: Routledge.

Susinos, T. (2012). Presentación. Las posibilidades de la voz del alumnado para el cambio y la mejora educativa. Revista de Educación, 359, 16-23.

Tharp, D. S. (2015). Using Critical Discourse Analysis to Understand Student Resistance to Diversity. Multicultural Education, 23 (1) 2-8.

Tójar J. C. (2006). Investigación cualitativa: Comprender y actuar. Madrid: La Muralla.

Tomlinson, C. (2003). Fulfilling the promise of the differentiated classroom: Strategies and tools for responsive teaching. Alexandria, VA: Association for Supervision and Curriculum Development.

Topping, K., Buchs, C., Duran, D., \& Van Keer, H. (2017). Effective peer learning: From principles to practical implementation. Londres: Routledge.

Zabalza, M.A. (1990). Fundamentación de la Didáctica y del conocimiento. En A. Medina y M.L. Sevillano, Didáctica. (pp. 85-220). Madrid: UNED.

Fecha de recepción: 6 de mayo de 2017

Fecha de revisión: 15 de mayo de 2017

Fecha de aceptación: 12 de marzo de 2018 
